\title{
EMOTION DETECTION FROM TEXT DOCUMENTS
}

\author{
Shiv Naresh Shivhare and Sri Khetwat Saritha \\ Department of CSE and IT, Maulana Azad National Institute of Technology, \\ Bhopal, Madhya Pradesh, India
}

\begin{abstract}
Emotion Detection is one of the most emerging issues in human computer interaction. A sufficient amount of work has been done by researchers to detect emotions from facial and audio information whereas recognizing emotions from textual data is still a fresh and hot research area. This paper presented a knowledge based survey on emotion detection based on textual data and the methods used for this purpose. At the next step paper also proposed a new architecture for recognizing emotions from text document. Proposed architecture is composed of two main parts, emotion ontology and emotion detector algorithm. Proposed emotion detector system takes a text document and the emotion ontology as inputs and produces one of the six emotion classes (i.e. love, joy, anger, sadness, fear and surprise) as the output.
\end{abstract}

\section{KEYWORDS}

Textual Emotion Detection; Emotion Word Ontology; Human-Computer Interaction

\section{INTRODUCTION}

Detecting emotional state of a person by analyzing a text document written by him/her appear challenging but also essential many times due to the fact that most of the times textual expressions are not only direct using emotion words but also result from the interpretation of the meaning of concepts and interaction of concepts which are described in the text document. Recognizing the emotion of the text plays a key role in the human-computer interaction [1]. Emotions may be expressed by a person's speech, face expression and written text known as speech, facial and text based emotion respectively. Sufficient amount of work has been done regarding to speech and facial emotion recognition but text based emotion recognition system still needs attraction of researchers [14]. In computational linguistics, the detection of human emotions in text is becoming increasingly important from an applicative point of view.

The paper is organized as following. Section 2 describes the methods used for text based emotion detection, which is classified into keyword spotting technique, lexical affinity method, learning based method and hybrid approach along with the limitations of these existing methods. A proposed architecture which contains the emotion ontology and emotion detector algorithm is explained in Section 3. Based on this, a system is designed for emotion detection from text documents. Finally conclusion is given in Section 4.

\section{RELATED WORK}

The concept of affective computing in 1997 by Since Picard [3] proposed that the role of emotions in human computer interaction. This domain attracted many researchers from computer 
International Journal of Data Mining \& Knowledge Management Process (IJDKP) Vol.4, No.6, November 2014

science, biotechnology, psychology, and cognitive science and so on. Following the trend, the research in the field of emotion detection from textual data emerged to determine human emotions from another point of view. Problem of emotion recognition from text can be formulated as follows: Let $\mathrm{E}$ be the set of all emotions, $\mathrm{A}$ be the set of all authors, and let $\mathrm{T}$ be the set of all possible representations of emotion-expressing texts. Let $r$ be a function to reflect emotion e of author a from text t, i.e., $\mathrm{r}$ : $\mathrm{A} x \mathrm{~T} \rightarrow \mathrm{E}$, then the function $\mathrm{r}$ would be the answer to the problem [4].

The main problem of emotion recognition systems lies in fact that, although the definitions of $\mathrm{E}$ and $\mathrm{T}$ may be straightforward, the definitions of individual element, even subsets in both sets of $\mathrm{E}$ and $\mathrm{T}$ would be rather confusing. On one side, for the set $\mathrm{T}$, new elements may add in as the languages are constantly emerging. Whereas on the other side, currently there are no standard classifications of "all human emotions" due to the complex nature of human minds, and any emotion classifications can only be seen as "labels" annotated afterwards for different purposes. Methods used for text based emotion recognition system [4], [5] are:

\subsection{Keyword Spotting Technique}

The keyword spotting technique can be described as the problem of finding occurrences of keywords (emotion words here) from a given text document. This problem has been studied in the past and algorithms have been suggested for solving it. In the context of emotion detection this method is based on certain predefined keywords. These words are classified into categories such as disgusted, sad, happy, angry, fearful, surprised etc. Occurrences of these emotion words find and on the basis of that an emotion class is assigned to the text document.

\subsection{Lexical Affinity Method}

Detecting emotions based on related keywords is an easy to use and straightforward method. Lexical Affinity approach is an extension of keyword spotting technique. It assigns a probabilistic 'affinity' for a particular emotion to arbitrary words apart from picking up emotional keywords. These probabilities are often part of linguistic corpora but have disadvantages; firstly the assigned probabilities are biased toward corpus-specific genre of texts, secondly it misses out emotional content that resides deeper than the word-level on which this technique operates e.g. the word 'accident' having been assigned a high probability of indicating a negative emotion, would not contribute correctly to the emotional assessment of phrases like 'I avoided an accident' or 'I met my girlfriend by accident'.

\subsection{Learning-based Methods}

Learning-based methods are being used to formulate the problem differently. Originally the problem was to determine emotions from input texts but now the problem is to classify the input texts into different emotions. Unlike keyword-based detection methods, learning-based methods try to detect emotions based on a previously trained classifier, which apply various theories of machine learning such as support vector machines [8] and conditional random fields [9], to determine which emotion category should the input text belongs.

\subsection{Hybrid Methods}

Since keyword-based methods with thesaurus and naïve learning-based methods could not acquire satisfactory results, some systems use hybrid approach by combining both keyword spotting technique and learning based method, which helps to improve accuracy. 


\subsection{Limitations}

From above discussion there are few limitations [7]:

\subsubsection{Ambiguity in Keyword Definitions}

Using emotion keywords is a straightforward way to detect associated emotions, the meanings of keywords could be multiple and vague, as most of the words could change their meanings according to different usages and contexts. Moreover, even the minimum set of emotion labels (without all their synonyms) could have different emotions in some extreme cases such as ironic or cynical sentences.

\subsubsection{Incapability of Recognizing Sentences without Keywords}

Keyword-based approach is totally based on the set of emotion keywords. Therefore, sentences without any keyword would imply that they do not contain any emotion at all, which is obviously wrong e.g. "I passed my qualify exam today" and "Hooray! I passed my qualify exam today" should imply the same emotion (joy), but the former sentence without "hooray" could remain undetected if "hooray" is the only keyword to detect this emotion.

\subsubsection{Lack of Linguistic Information}

Syntax structures and semantics also have influences on expressed emotions. For example, "I laughed at him" and "He laughed at me" would suggest different emotions from the first person's perspective. As a result, ignoring linguistic information also poses a problem to keyword-based methods.

\subsubsection{Difficulties in Determining Emotion Indicators [10]}

Learning-based methods can automatically determine the probabilities between features and emotions but the methods still need keywords in the form of features. The most intuitive features may be emoticons which can be seen as author's emotion annotations in the texts. The cascading problems would be the same as those in keyword-based methods.

\section{PROPOSED ARCHITECTURE}

The proposed architecture is very simple and easy to understand. This model is based on keyword spotting technique apart from that it also uses the concept of ontology. Use of ontology makes this model more efficient than other methods in recognizing emotions from text input.

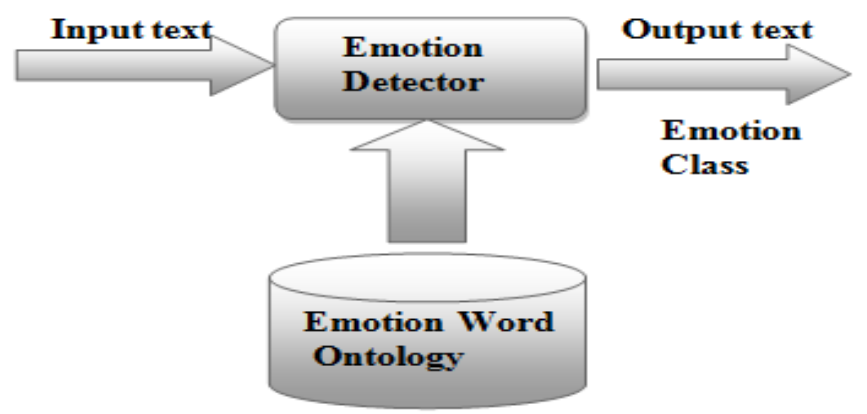

Figure 1. Proposed Architecture 
The Framework is divided into two main components: Emotion Ontology, Emotion Detector.

\subsection{Emotion Ontology}

Ontology is an explicit specification of conceptualization. Ontologies have definitional aspects like high level schemas and aspects like entities and attributes interrelationship is between entities, domain vocabulary [16]. Ontology allows a programmer to specify, in an open, meaningful way the concepts and relationships that collectively characterise some domain.

Emotion is expressed as joy, sadness, anger, surprise, hate, fear and so on. Since there is not any standard emotion word hierarchy, focus is on the related research about emotion in cognitive psychology domain. In 2001, W. Gerrod Parrot [2], wrote a book named "Emotions in Social Psychology", in which he explained the emotion system and formally classified the human emotions through an emotion hierarchy in six classes at primary level which are Love, Joy, Anger, Sadness, Fear and Surprise. Certain other words also fall in secondary and tertiary levels. This emotion word hierarchy is converted into ontology. Proposed ontology has class and subclass relationship format. Emotion classes at the primary level in emotion hierarchy are at the top of emotion ontology and emotion classes at the tertiary level are at the bottom of ontology. Emotion ontology is developed by Protégé (a free, open source ontology editor and knowledgebase framework).

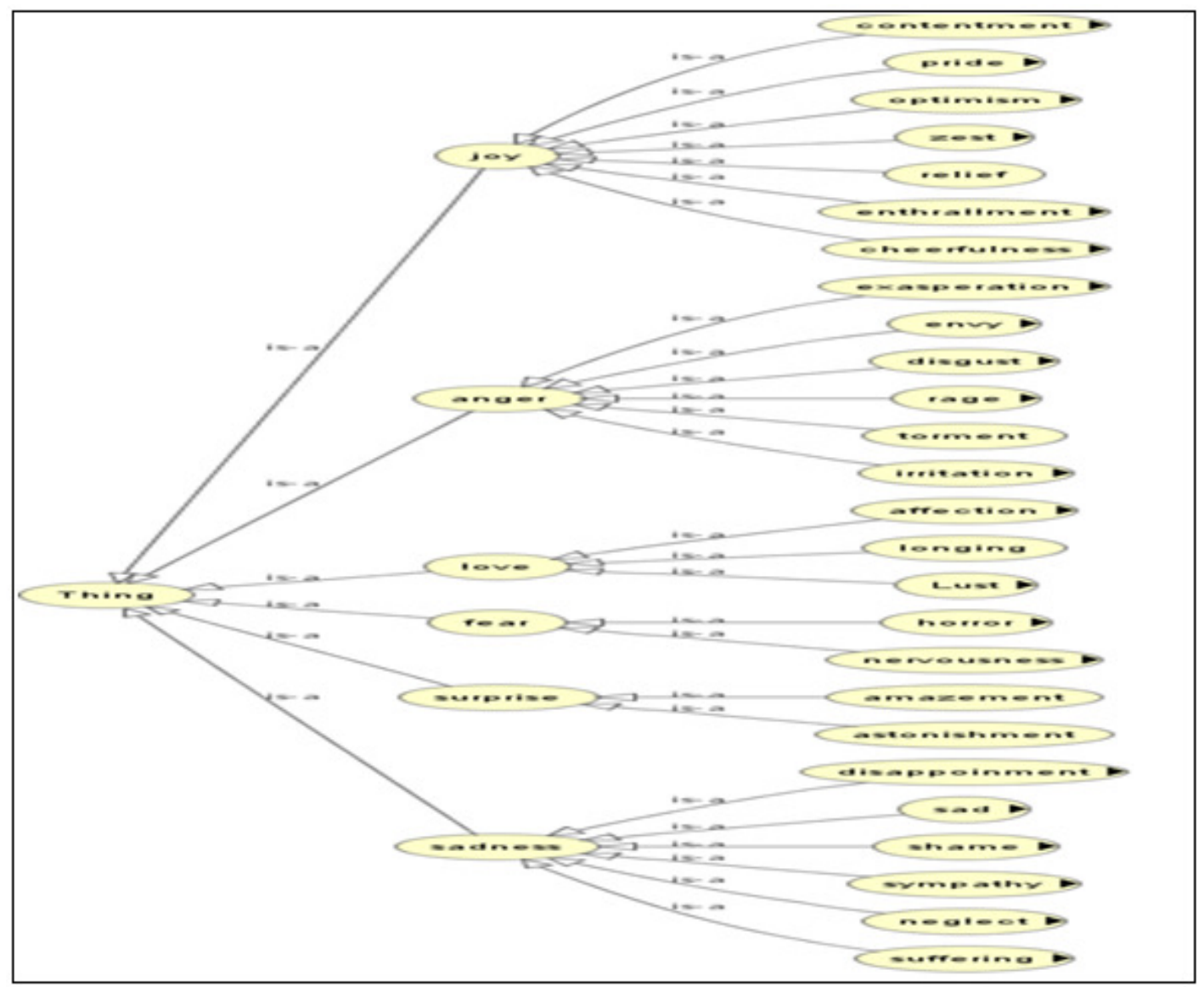

Figure 2. Emotion Ontology

Emotion ontology is an ontology representing "emotion" domain. A schematic diagram of emotion ontology is shown above in figure 2. Emotion ontology is displayed in left to right order. 
Only two levels (primary and secondary) are displayed in the image of emotion ontology while the tertiary level is hidden. Every emotion word in the ontology is an emotion class. "Thing" is the root and the super most class of the ontology. Every child class is related to its parent class by "is-a" relationship (child is-a parent) e.g. "anger" is a "thing" and "pride" is a "joy".

\subsection{Emotion Detector Algorithm}

Emotion of the text document can be recognized with the help of this emotion detector algorithm. The algorithm calculates score for every emotion class of primary level available in the emotion ontology by adding the scores of its respective secondary and tertiary levels' emotion classes. In final step scores of all the primary level classes are compared and emotion class having maximum score will be declared as the "emotion" of the input text document.

\subsubsection{Parameters Used}

Algorithm calculates score for all the emotion words in the emotion ontology so that they could be compared according to it. Certain parameters are required for this purpose. Task of calculating the parameters can be achieved with the help of Jena library which allows traversal and parsing of ontology. Required parameters are as follows:

\subsubsection{Parent-Child relationship}

If a text document belongs to a child; it also indirectly refers to the parent of it. Hence if a certain value is added to the child's score, parent score also need to be modified. This is achieved by traversing the ontology model in a breadth first manner using Jena API. When any node is encountered all of its children are retrieved. Then same method is applied to every child.

\subsubsection{Depth in Ontology}

Depth is required as it gives an idea about how specific is the term in relation to its corresponding ontology structure. The more specific it is the more weight age should be given to it. This value is calculated simultaneously while traversing the ontology tree.

\subsubsection{Frequency in Text document}

An emotion class will be more important if the same emotion word exists multiple times in the input text document. Frequency is an important parameter as more is the frequency more will be the importance of that term. Frequency of emotion words is calculated by parsing the text document and searching for occurrences of emotion words of emotion ontology.

\subsubsection{Algorithm}

Following algorithm is proposed to calculate the score for each emotion word of emotion ontology with the help of parameters from previous step. This score will be directly proportional to the frequency of the term and inversely proportional to its depth in the ontology. Hence a formula devised for the $\mathrm{m}^{\text {th }}$ terminology. Algorithm is as follows:

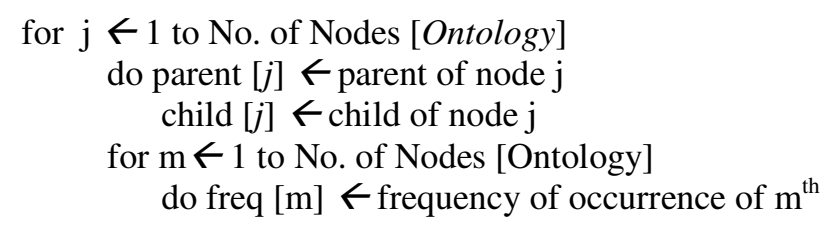


International Journal of Data Mining \& Knowledge Management Process (IJDKP) Vol.4, No.6, November 2014

$$
\text { depth }[\mathrm{m}] \leftarrow \text { depth of } \mathrm{m}^{\text {th }} \text { node in ontology }
$$

\section{Calculate (x):}

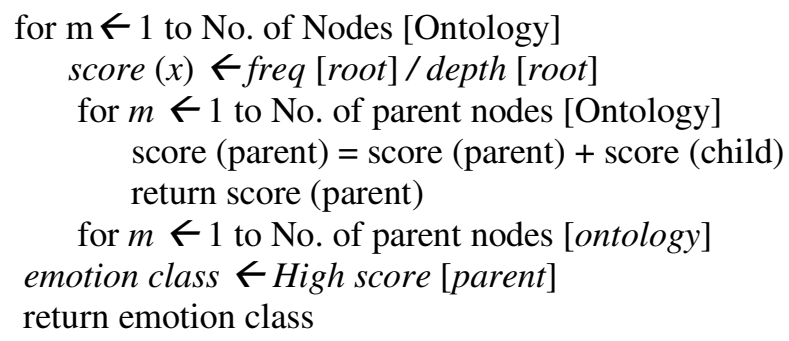

Where Nodes [Ontology] denotes emotion classes (emotion words) in the ontology, Parent [j] denotes parent classes in the ontology, Child [j] denotes child classes in the ontology, Freq [m] denotes frequency of $\mathrm{m}^{\text {th }}$ class in text document, Depth denotes depth of particular class in emotion ontology starting from the root class, Score [parent] denotes score of parent class in emotion ontology.

By proposed algorithm we find out the score of primary level emotion classes. Primary level emotion class having highest score will be decided as the final "emotion" for input text document.

\section{CONCLUSION}

In the era of web 2.0, text-based input is the most common way for humans to interact with computers, and thus emotion detection from text should be focused as an important research issue in affective computing.

In this paper, existing research of emotion detection based on textual data is surveyed and limitations of existing methods are reviewed. System architecture is proposed to improve detection capabilities and perform the task efficiently. Proposed system is based on keyword spotting technique as well as having rich features of ontology. Not all the limitations of existing methods are overcome by this architecture but use of ontology improves the detection capability by applying semantic approach.

\section{REFERENCES}

[1] R. Cowie, E. Douglas-Cowie, N. Tsapatsoulis, G. Votsis, S. Kollias, "Emotion recognition in humancomputer interaction,” in IEEE Signal Processing Magazine, vol. 18(1), Jan. 2001, pp. 32-80, doi: 10.1109/79.911197

[2] Parrott, W.G, "Emotions in Social Psychology," in Psychology Press, Philadelphia 2001

[3] C. Maaoui, A. Pruski, and F. Abdat, "Emotion recognition for human machine communication", Proc. IEEE/RSJ International Conference on Intelligent Robots and Systems (IROS 08), IEEE Computer Society, Sep. 2008, pp. 1210-1215, doi: 10.1109/IROS.2008.4650870

[4] Chun-Chieh Liu, Ting-Hao Yang, Chang-Tai Hsieh, Von-Wun Soo, "Towards Text-based Emotion Detection: A Survey and Possible Improvements ",in International Conference on Information Management and Engineering,2009.

[5] N. Fragopanagos, J.G. Taylor, "Emotion recognition in human-computer interaction", Department of Mathematics, King's College, Strand, London WC2 R2LS, UK Neural Networks 18 (2005) 389-405 march 2005.

[6] C. Elliott, "The affective reasoner: a process model of emotions in a multiagent system," in Doctoral thesis, Northwestern University, Evanston, IL, May 1992. 
International Journal of Data Mining \& Knowledge Management Process (IJDKP) Vol.4, No.6, November 2014

[7] C.-H. Wu, Z.-J. Chuang, and Y.-C. Lin, "Emotion Recognition from Text Using Semantic Labels and Separable Mixture Models," ACM Transactions on Asian Language Information Processing (TALIP), vol. 5, issue 2, Jun. 2006, pp. 165-183, doi:10.1145/1165255.1165259.

[8] Z. Teng, F. Ren, and S. Kuroiwa, "Recognition of Emotion with SVMs," in Lecture Notes of Artificial Intelligence 4114, D.-S. Huang, K. Li, and G. W. Irwin, Eds. Springer, Berlin Heidelberg, 2006, pp. 701-710, doi: 10.1007/11816171_87.

[9] C. Yang, K. H.-Y. Lin and H.-H. Chen, "Emotion classification using web blog corpora," Proc. IEEE/WIC/ACM International Conference on Web Intelligence. IEEE Computer Society, Nov. 2007, pp. 275-278, doi: 10.1109/WI.2007.50.

[10] C. M. Lee, S. S. Narayanan, and R. Pieraccini, "Combining Acoustic and Language Information for Emotion Recognition," Proc. 7th International Conference on Spoken Language Processing (ICSLP02), 2002, pp.873-876.

[11] C.-H. Wu, Z.-J. Chuang and Y.-C. Lin, "Emotion Recognition from Text Using Semantic Labels and Separable Mixture Models," ACM Transactions on Asian Language Information Processing (TALIP), vol. 5, issue 2, Jun. 2006, pp. 165-183, doi:10.1145/1165255.1165259.

[12] C. Elliott, "The affective reasoner: a process model of emotions in a multiagent system," in Doctoral thesis, Northwestern University, Evanston, IL, May 1992

[13] Protégé tool, www.protege.stanford.edu/,

[14] Nicu Sebea, Ira Cohenb, Theo Geversa, and Thomas S. Huangc "Multimodal Approaches for Emotion Recognition: A Survey", USA

[15] http://sail.usc.edu/ kazemzad/emotion_in_text_cgi/DAL_app/index.php?overall=bad\&submit_evalua tion=Submit+Query".

[16] http://www.wikipedia.org/ 